\title{
Ground-state energy of the $d=1,2,3$ dimensional Hubbard model in the weak-coupling limit
}

\author{
Walter Metzner and Dieter Vollhardt \\ Institut für Theoretische Physik C, Technische Hochschule Aachen, D-5100 Federal Republic of Germany
}

(Received 26 May 1988)

\begin{abstract}
The correlation energy of the Hubbard model on simple-cubic lattices is calculated in the weakcoupling regime for dimensions $d=1,2,3$. It is shown that, in spite of the nonanalytic dependences known to arise and in contrast to earlier expectations, the exact second-order contribution to the ground-state energy in $d=1$ can be obtained by ordinary perturbation theory. This result is argued to hold for any finite order and, in particular, for any dimension.
\end{abstract}

\section{INTRODUCTION}

In spite of the apparent simplicity of the Hubbard model ${ }^{1-3}$ and 25 years of research only few reliable analytic results exist. In one dimension $(d=1)$ the exact result for the ground-state energy in the case of nextneighbor hopping has been obtained by Lieb and $\mathrm{Wu}^{4}$ using the Bethe ansatz. At half-filling the energy is a nonanalytic function of the interaction strength $U$ for $U \rightarrow 0$, owing to antiferromagnetic correlations which exist for all $U>0$. The latter are typical for $A B$-type lattices in arbitrary dimensions and arise from the "perfect nesting" property of the Fermi surface in the case of nextneighbor hopping. ${ }^{5}$ Hence, for such lattices even the weak-coupling regime of the Hubbard model is nontrivial. Nevertheless, in this paper we show that - in contrast to results obtained earlier ${ }^{6}$ - the correlation energy of the Hubbard model in $d=1$ in the weak-coupling limit can be obtained exactly by standard diagrammatic perturbation theory. Calculating the corresponding expression in $d=2$ and $d=3$ we conjecture that this holds for any dimension and for any order. A rigorous mathematical proof for this conjecture still does not exist.

\section{THE HUBBARD MODEL}

The single-band Hubbard model has the form

$$
\begin{aligned}
H & =H_{0}+H_{1}, \\
H_{0} & =\sum_{i j, \sigma} t_{i j} c_{i \sigma}^{\dagger} c_{j \sigma} \\
& =\sum_{\mathbf{k}, \sigma} \varepsilon_{\mathbf{k}} a_{\mathbf{k} \sigma}^{\dagger} a_{\mathbf{k} \sigma}, \\
H_{1} & =U \sum_{j} n_{j \uparrow} n_{j \downarrow} \\
& =\frac{U}{L} \sum_{\mathbf{k}, \mathbf{k}^{\prime}, q} a_{\mathbf{k}+q, \uparrow}^{\dagger} a_{\mathbf{k} \uparrow} a_{\mathbf{k}^{\prime}-q, \downarrow}^{\dagger} a_{\mathbf{k}^{\prime} \downarrow},
\end{aligned}
$$

where $H_{0}$ is the kinetic (hopping) energy, $H_{1}$ is the onsite interaction $(U>0)$, and $c_{i \sigma}^{\dagger}\left(c_{i \sigma}\right)$ are creation (annihilation) operators for spins $\sigma$ on site $i$ on a $d$-dimensional lattice, whose corresponding counterparts in $\mathbf{k}$ space are given by $a_{\mathrm{k} \sigma}^{\dagger}\left(a_{\mathbf{k} \sigma)}\right.$. The respective number operators are given by $n_{i \sigma}=c_{i \sigma}^{\dagger} c_{i \sigma}$ and $n_{\mathrm{k} \sigma}=a_{\mathrm{k} \sigma}^{\dagger} a_{\mathrm{k} \sigma}$. We consider a fixed number $N_{\sigma}$ of particles with spin $\sigma$ on a lattice with $L$ sites, with densities given by $n_{\sigma}=N_{\sigma} / L, n=n_{\uparrow}+n_{\downarrow}$. We always take $n_{\sigma} \leq \frac{1}{2}$, since the case $\frac{1}{2}<n_{\sigma} \leq 1$ is obtained from a particle-hole transformation.

In $d=1$ and for next-neighbor hopping the groundstate energy $E\left(n_{\uparrow}, n_{\downarrow}, U\right)$ has been calculated exactly. ${ }^{4}$ For general densities $n_{\uparrow}, n_{\downarrow}$ it is given implicitly by an integral equation. In the special case $n_{\uparrow}=n_{\downarrow}=\frac{1}{2}$ (halffilled band) this leads to an explicit expression ${ }^{4}$

$$
E\left(\frac{1}{2}, \frac{1}{2}, U\right)=-4 t I(U / 4 t),
$$

where

$$
I(z)=\int_{0}^{\infty} d \omega \frac{J_{0}(\omega) J_{1}(\omega)}{\omega[1+\exp (2 z \omega)]}
$$

and $J_{0}, J_{1}$ are Bessel functions. The analytic properties of $I(z)$ in the complex $z$ plane have been investigated in detail by Takahashi. ${ }^{7}$ Expressing (5) as a sum over hypergeometric functions he proved in particular that $I(z)$ is an infinitely many-valued function of $z$. There are logarithmic branch points at $z= \pm i / m, m=1,2,3 \ldots$. Hence, $z=0$ is the accumulation point of these branch points. Consequently, $E\left(\frac{1}{2}, \frac{1}{2}, U\right)$ cannot be represented by a Taylor series around $U=0$. Using this sum over hypergeometric functions Economou and Poulopoulos ${ }^{6}$ subsequently showed that $I(z)$ can be expanded in an asymptotic series

$$
I(z)=\frac{1}{\pi}-\frac{z}{4}+\sum_{m=1}^{N} \mu_{m} z^{2 m}+O\left(z^{2 N+2}\right),
$$

where

$\mu_{m}=\frac{(2 m-1)\left(2^{2 m+1}-1\right)[(2 m-3) ! !]^{3}}{2^{3 m-1}(m-1) !} \frac{\zeta(2 m+1)}{\pi^{2 m+1}}$

with $(-1) ! ! \equiv 1$ and $\zeta$ is the Riemann zeta function. The existence of such an expansion is in contrast to a proposition of Takahashi ${ }^{7}$ who asserted that $\partial^{3} E\left(\frac{1}{2}, \frac{1}{2}, U\right) / \partial U^{3}$ diverges in the limit $U \rightarrow 0^{+}$. However, this divergence is an artifact caused by an improper interchange of the limit $U \rightarrow 0^{+}$and the $\omega$ integral. Using (4) and (5) we calculated $\partial^{3} E\left(\frac{1}{2}, \frac{1}{2}, U\right) / \partial U^{3}$ numerically and found that it vanishes linearly in $U$, the slope being given by the fourth-order coefficient in (6). Similarly, calculating 
$\partial E / \partial U$ numerically the second-order coefficient in (6) came out correctly, too.

We note in passing that the existence of the asymptotic expansion (6) rules out contributions to $E$ of the form $U^{\alpha}(\ln U)^{\beta}$. On the other hand, exponentially small terms of the form $U^{\alpha} \exp (-t / U)$ connected with the (antiferromagnetic) instability of the ground state cannot be described by (6).

The asymptotic expansion in $d=1,(6)$, was derived from the already existing exact solution. The question is now whether (6) can also be obtained by other means, such as standard perturbation theory for the Hubbard Hamiltonian. If so, the same technique could also be applied in higher dimensions where exact results for the ground-state energy are not available.

\section{LEADING ORDER CORRELATION ENERGY}

The ground-state energy of the Hubbard model can be written as

$E\left(n_{\uparrow}, n_{\downarrow}, U\right)=E_{0}\left(n_{\uparrow}, n_{\downarrow}\right)+U n_{\uparrow} n_{\downarrow}+E_{c}\left(n_{\uparrow}, n_{\downarrow}, U\right)$,

where $E_{0}$ is the ground-state energy for the noninteracting case $(U=0), U n_{\uparrow} n_{\downarrow}$ is the first order correction (i.e., $E_{0}+U n_{\uparrow} n_{\downarrow}$ is the naive Hartree-Fock result) and $E_{c}$ is referred to as "correlation energy."

We will now put forward, and then investigate, the following conjectures.

(i) For small $U$ the energy $E_{c}\left(n_{\uparrow}, n_{\downarrow}, U\right)$ is of order $U^{2}$.

(ii) The coefficient of the $U^{2}$ term is produced correctly by employing standard Goldstone perturbation theory on the Hubbard Hamiltonian (1).
In $d=1$ the first conjecture is indeed proved to be correct: for $n_{\uparrow}=n_{\downarrow}=\frac{1}{2}$ an asymptotic expansion, (6), even exists, while for $n_{\uparrow}=n_{\downarrow}<\frac{1}{2}$ we checked it numerically by calculating $E_{c}$ for small $U$ from the Lieb-Wu integral equations; ${ }^{4,8}$ for $n_{\uparrow} \neq n_{\downarrow} E_{c}$ is analytic in $U=0 .{ }^{7}$

The perturbation expansion of the ground-state energy of the interacting system is given by ${ }^{9}$

$$
E=E_{0}+\left\langle\phi_{0}\left|H_{1} \sum_{j=0}^{\infty}\left[\frac{1}{E_{0}-H_{0}} H_{1}\right]^{j}\right| \phi_{0}\right\rangle_{c} .
$$

Here $H_{0}$ is the free Hamiltonian, $\left|\phi_{0}\right\rangle$ is the ground state and $E_{0}$ is the ground-state energy of the noninteracting system; $H_{1}$ is the perturbation. The matrix elements can be represented by the Goldstone diagrams. ${ }^{9}$ The subscript " $c$ " indicates that only connected diagrams must be included.

For the Hubbard model $H_{0}$ and $H_{1}$ are given by (2) and (3) respectively. The noninteracting ground state $\left|\phi_{0}\right\rangle$ is a Fermi sea of Bloch waves

$$
\left|\phi_{0}\right\rangle=\prod_{k \leq k_{F \uparrow}} a_{\mathbf{k} \uparrow}^{\dagger} \prod_{k^{\prime} \leq k_{F \downarrow}} a_{\mathbf{k}^{\prime} \downarrow}^{\dagger}|0\rangle
$$

where $|0\rangle$ is the vacuum state.

The first-order contribution is immediately obtained as $E_{1}=\left\langle\phi_{0}\left|H_{1}\right| \phi_{0}\right\rangle=L U n_{\uparrow} n_{\downarrow}$. In second order we have to calculate the diagram in Fig. 1. Usually there are two second-order diagrams; however, for the Hubbard interaction only one of them contributes. Using the rules for evaluating Goldstone diagrams ${ }^{10}$ we find

$$
E_{2}=\frac{L U^{2}}{(2 \pi)^{3 d}} \int_{\mathrm{BZ}} d \mathbf{k} d \mathbf{k}^{\prime} d \mathbf{q} \frac{n_{\mathbf{k} \uparrow}^{0} n_{\mathbf{k}^{\prime} \downarrow}^{0}\left(1-n_{\mathbf{k}+\mathbf{q} \uparrow}^{0}\right)\left(1-n_{\mathbf{k}^{\prime}-\mathbf{q} \downarrow}^{0}\right)}{\varepsilon_{\mathbf{k}}+\varepsilon_{\mathbf{k}^{\prime}}-\varepsilon_{\mathbf{k}+\mathbf{q}}-\varepsilon_{\mathbf{k}^{\prime}-\mathbf{q}}}
$$

The $\mathbf{k}, \mathbf{k}^{\prime}, \mathbf{q}$ integrations extend over the first Brillouin zone; $\mathbf{k}+\mathbf{q}$ and $\mathbf{k}^{\prime}-\mathbf{q}$ are defined up to reciprocal lattice vectors, i.e., Umklapp processes must be included; $n_{\mathbf{k} \sigma}^{0}$ is the k-space occupation of the noninteracting system.

For $d=1$ and next-neighbor hopping we have $\varepsilon_{\mathrm{k}}=-2 t \cos k$, where the lattice constant $a \equiv 1$. In this

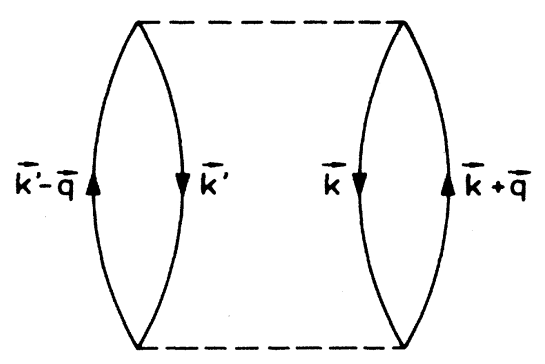

FIG. 1. Second-order Goldstone diagram. case $E_{2}$ can be calculated analytically for $n_{\uparrow}=n_{\downarrow}=\frac{1}{2}$ (see the Appendix). The result is

$$
E_{2} / L=-\frac{7}{16 \pi^{3}} \xi(3) \frac{U^{2}}{t}
$$

in agreement with Eqs. (4) and (6). Thus, in this case perturbation theory yields the exact asymptotic result, i.e., the second conjecture is also confirmed. This amends an earlier result, ${ }^{6}$ which was obtained numerically from an incorrect expression for the second-order perturbation contribution $E_{2}$. For $n_{\uparrow}=n_{\downarrow}<\frac{1}{2}$ we have evaluated the integral in Eq. (11) numerically. Again the result coincides with the exact asymptotic $U^{2}$ contribution as obtained by numerical integration of the Lieb-Wu equations. We note that at half-filling $E_{2}$ approximates $E_{c}$ very well (error $<10 \%$ ) even for intermediate coupling strengths $(U / t \leq 10)$. In this regime the fourth-order contribution is no more negligible and is partially compensated by the remaining nonanalytical contributions.

Encouraged by these results we used Eq. (11) to calcu- 


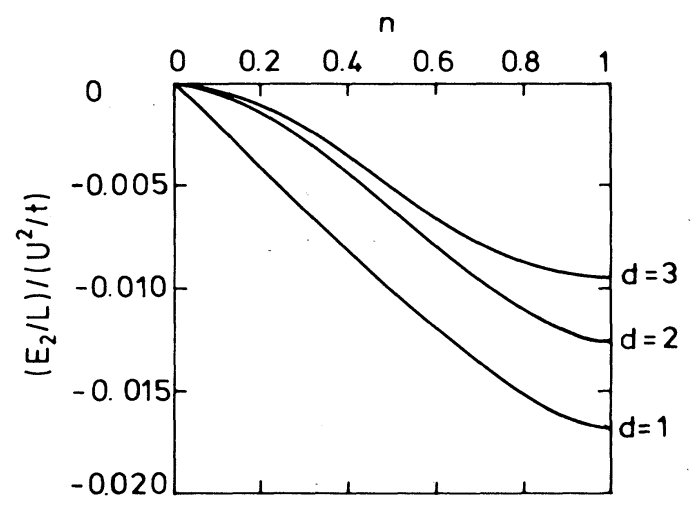

FIG. 2. Second-order correlation energy as a function of the density $n$ for various lattice dimensions $d$.

late $E_{2}$ also for higher dimensional lattices, i.e., on a square lattice in $d=2$ and a simple-cubic lattice in $d=3,{ }^{11}$ where for next-neighbor hopping

$$
\varepsilon_{\mathrm{k}}=-2 t \sum_{j=1}^{d} \cos k_{j} .
$$

This energy dispersion has the perfect-nesting property $\varepsilon_{\mathbf{k}}=-\varepsilon_{\mathbf{k}}+\mathbf{Q} / 2$ where $\mathbf{Q}$ is a reciprocal-lattice vector. This implies that for $U>0$ antiferromagnetic correlations exist in any dimension, ${ }^{5}$ leading to nonanalytic contributions to the ground-state energy for small $U$. Hence as in $d=1$, a Taylor expansion around $U=0$ is ruled out, but an asymptotic expansion is not affected by such exponentially small terms. For $d=2$ and 3 the integral (11) has been evaluated by Monte Carlo techniques. In Fig. 2 we show $E_{2}$ as a function of $n\left(n_{\uparrow}=n_{\downarrow}\right)$. For $d=2,3$ the correlation energy is proportional to $n^{2}$ for small densities. In $d=1$ we find $E_{2} \propto n$ for small $n$. This is due to the algebraic van Hove singularity in the density of states at the minimum of $\varepsilon_{\mathrm{k}}$ in one dimension.

A calculation of $E_{2}$ is also possible in $d=\infty$, where the evaluation of the integral (11) becomes particularly simple. Note that $E_{2}$ scales like $d^{-1 / 2}$ for large dimensions and fixed hopping amplitude $t$. Hence $E_{2} \cdot d^{1 / 2}$ is a finite quantity in $d=\infty$. The result yields a very good approximation of that in $d=3$ and will be published elsewhere ${ }^{12}$ together with a presentation of the method of how to calculate in $d=\infty$ and other results for the Hubbard model in $d=\infty$.

\section{HIGHER ORDERS}

The above results suggest that perturbation theory also yields the higher-order corrections in $U$. To investigate this we calculated the fourth-order perturbation contribution for $n_{\uparrow}=n_{\downarrow}=\frac{1}{2}$ in $d=1$ (the odd-order terms are zero, due to particle-hole symmetry) and compared with (6). The evaluation of the relevant Goldstone diagrams, ${ }^{13}$ altogether 15 diagrams, was carried out numerically by a Monte Carlo technique. Summing up all the diagrams one obtains a coefficient for the $U^{4}$ correction to the ground-state energy which agrees with the exact coefficient in (6) within numerical accuracy. ${ }^{14}$ Since $n_{\uparrow}=n_{\downarrow}=\frac{1}{2}$ is the most delicate case [there is a cusp in the ground-state energy as a function of $n_{\uparrow}, n_{\downarrow}$ in $n_{\uparrow}=n_{\downarrow}=\frac{1}{2}$ for any nonzero $U$ (Ref. 4)] it is plausible to conclude that perturbation theory yields the correct $U^{4}$ term for all $n_{\uparrow}, n_{\downarrow}$.

In sixth order some of the Goldstone diagrams are divergent. Consider, for instance, the diagram shown in Fig. 3(a) in $d=1$ for simplicity (a generalization of the subsequent discussion to arbitrary $d$ is straightforward). The intermediate state $a_{k+q, \sigma}^{\dagger} a_{k \sigma} a_{k^{\prime}-q,-\sigma}^{\dagger} a_{k,,^{\prime}-\sigma}\left|\phi_{0}\right\rangle$ occurs three times, giving rise to a factor $\left(\varepsilon_{k}-\varepsilon_{k+q}\right.$ $\left.+\varepsilon_{k^{\prime}}-\varepsilon_{k^{\prime}-q}\right)^{-3}$. The corresponding excitation energy $\varepsilon=\varepsilon_{k}-\varepsilon_{k+q}+\varepsilon_{k^{\prime}}-\varepsilon_{k^{\prime}-q}$ vanishes for

$$
q=0, \quad k=\mp k_{F \sigma}, \quad k^{\prime}= \pm k_{F-\sigma}
$$

and, if $k_{F \uparrow}=k_{F \downarrow}=k_{F}$ (i.e., $n_{\uparrow}=n_{\downarrow}$ ), for

$$
q= \pm 2 k_{F}, \quad k=\mp k_{F}, \quad k^{\prime}= \pm k_{F}
$$

For small deviations of $\left(q, k, k^{\prime}\right)$, with $|k|<k_{F \sigma}$, $\left|k^{\prime}\right|<k_{F,-\sigma},|k+q|>k_{F \sigma},\left|k^{\prime}-q\right|>k_{F,-\sigma}$, from a point $\left(q_{0}, k_{0}, k_{0}^{\prime}\right)$ for which $\varepsilon$ vanishes, the excitation energy $\varepsilon$ is proportional to

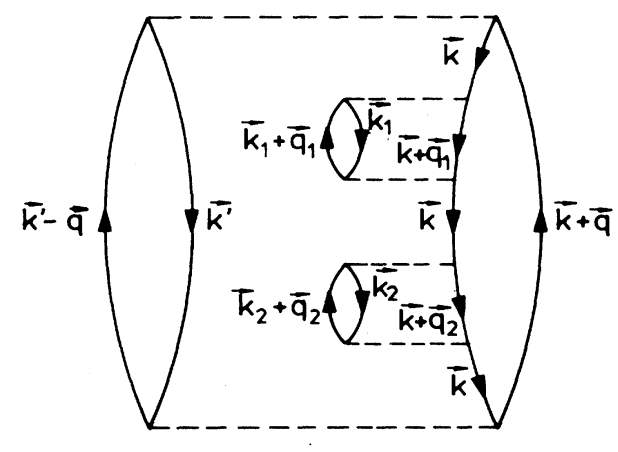

(a)

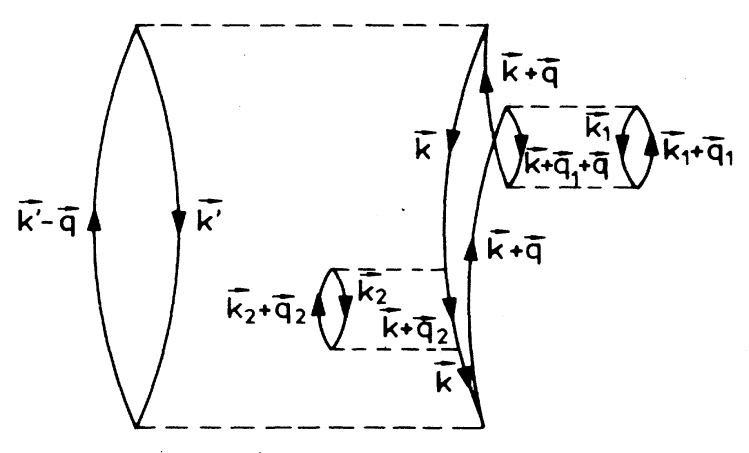

(b)

FIG. 3. Divergent sixth-order diagrams. 


$$
\delta \equiv\left[\left(q-q_{0}\right)^{2}+\left(k-k_{0}\right)^{2}+\left(k^{\prime}-k_{0}^{\prime}\right)^{2}\right]^{1 / 2} .
$$

The integration volume of a $\delta$ neighborhood of $\left(q_{0}, k_{0}, k_{0}^{\prime}\right)$ is proportional to $\delta^{3}$. Since $\varepsilon^{-3} \propto \delta^{-3}$ the $q, k, k^{\prime}$ integrations diverge logarithmically. Note that the factors corresponding to the other intermediate states

$a_{k_{i}+q_{i},-\sigma}^{\dagger} a_{k_{i},-\sigma} a_{k+q, \sigma}^{\dagger} a_{k+q_{i}, \sigma} a_{k^{\prime}-q,-\sigma}^{\dagger} a_{k^{\prime}-\sigma}\left|\phi_{0}\right\rangle$

are finite and nonzero in $\left(q_{0}, k_{0}, k_{0}^{\prime}\right)$. The diagram shown in Fig. 3(b) diverges too. This divergence is due to the same poles as in the graph in Fig. 3(a). The signs of the diagrams (determined by the number of loops and hole lines, see Ref. 10) are opposite. It is easy to see that the sum of both diagrams (including the signs) gives a convergent integral, i.e., the divergencies cancel each other. Collecting all divergent diagrams in sixth order one finds that their sum defines a convergent integral. Generally speaking, one obtains finite contributions in each finite order of perturbation theory if one performs the momentum integrals after having summed up all the corresponding diagrams. Of course, the sum over all orders in $U$ may nevertheless be divergent [the series in (6) is divergent for arbitrarily small $U$ ]. In view of the fact that perturbation theory is correct up to forth order in $U$ and finite in each finite order, we believe that perturbation theory reproduces the complete asymptotic expansion of the ground-state energy of the one-dimensional Hubbard model.

In higher dimensions $d=2,3, \ldots$ there is no exact result for the ground-state energy to compare with. However, as far as perturbation theory is concerned, there are no qualitative changes in $d>1$ : each other yields welldefined finite expressions. Hence, we expect that also in this case perturbation theory produces the weak-coupling limit of the correlation energy.

We finally remark that there are two different ways of defining the ground-state energy of an infinitely large system. One way is to simply take the lowest eigenvalue of the Hamiltonian in the thermodynamic limit. Alternatively, one may define the ground-state energy as the energy of an infinitely large system at temperature $T$, in the limit $T \rightarrow 0$. The Goldstone formula used in this paper is based on the first version. Kohn and Luttinger ${ }^{15}$ realized that the above definitions of the term "ground-state energy" are not generally equivalent. However, if the unperturbed energies of the particles and their interactions are spatially isotropic, both definitions yield the same energy. ${ }^{16}$ The one-dimensional Hubbard model is indeed isotropic. In second-order perturbation theory, the difference between both ground-state energies is ${ }^{15}$

$$
E_{2}^{\prime}=\lim _{T \rightarrow 0}\left(\Omega_{2}^{A}\left(\mu_{0}\right)-\frac{1}{2} \frac{\left[\partial \Omega_{1}^{G}\left(\mu_{0}\right) / \partial \mu_{0}\right]^{2}}{\partial^{2} \Omega_{0}\left(\mu_{0}\right) / \partial \mu_{0}^{2}}\right]
$$

where $\Omega_{0}$ denotes the unperturbed grand-canonical potential, $\Omega_{1}^{G}$ the first-order Goldstone contribution and $\Omega_{2}^{A}$ the second-order contribution of anomalous diagrams (not included in Goldstone's expansion), $\mu_{0}$ is the unperturbed chemical potential. For the Hubbard model $E_{2}^{\prime}$ is zero in arbitrary dimensions, as is easily seen by explicit calculation. Hence, at least up to second order in $U$ there is no ambiguity in the definition of the ground-state energy of the $d$-dimensional Hubbard model.

In actual experiments one can neither measure at zero temperature, nor is the system infinitely large. However, for typical applications of the Hubbard model in condensed matter physics the limit $T \rightarrow 0$ should be taken after performing the thermodynamic limit, since the level spacing of the unperturbed system is usually many orders of magnitude smaller than $k_{B} T$.

\section{CONCLUSION}

The perturbation expansion for the ground-state energy of the Hubbard model has been investigated. We have shown that perturbation theory yields finite contributions in each order in the coupling constant $U$. Comparing with the exact asymptotic expansion of the ground-state energy in $d=1$, we proved that perturbation theory describes the asymptotic behavior ( $U^{2}$ contribution) of the ground-state energy in $d=1$ correctly. We then calculated the correlation energy for higher dimensions in leading order of the coupling constant and conjectured that perturbation theory even yields the exact results in higher orders and, in particular, in higher dimensions. A rigorous proof of this conjecture would certainly be very interesting. Our results for the weak-coupling limit of the Hubbard model may be of relevance for current investigations of the ground-state properties of the twodimensional model in the context of high- $T_{c}$ superconductivity. ${ }^{17}$ Besides the fact that the weak-coupling regime is of interest by itself, e.g., in view of scaling approaches, ${ }^{18}$ our results allow for an explicit assessment of the small $U$ behavior of specific variational wave functions, which are thought to describe the ground state in the strong-coupling regime. ${ }^{12,17}$

\section{ACKNOWLEDGMENT}

We are grateful to F. Gebhard, Dr. P. van Dongen, and Professor G. Czycholl for valuable discussion.

\section{APPENDIX: CALCULATION OF $E_{2}$ FOR $d=1$ AND $n_{\uparrow}=n_{\downarrow}=\frac{1}{2}$}

For a one-dimensional lattice chain with lattice constant $a=1$ the first Brillouin zone (BZ) lies in the interval $[-\pi, \pi]$, furthermore $n_{k \sigma}^{0}=\Theta\left(k_{F \sigma}-|k|\right)$ where $k_{F \sigma}$ $=\pi n_{\sigma}=\pi / 2$ for $n_{\uparrow}=n_{\downarrow}=\frac{1}{2}$. We define

$$
n_{\sigma}^{0}(k, q)=n_{k \sigma}^{0}\left(1-n_{k+q, \sigma}^{0}\right)
$$

and

$$
\varepsilon(k, q)=\varepsilon_{k+q}-\varepsilon_{k},
$$

the latter being the excitation energy for an electron-hole pair. For next-neighbor hopping with amplitude $t=1$ we have $\varepsilon_{k}=-2 \cos k$ and thus $\varepsilon(k, q)=4 \sin (k+q$ / $2) \sin (q / 2)$. Inserting this into (11) and using inversion symmetry we find 


$$
E_{2} / L=-\frac{U^{2}}{(2 \pi)^{3}} \int_{0}^{\pi} \frac{d q}{2 \sin (q / 2)} \int_{-\pi}^{\pi} d k d k^{\prime} \frac{n_{\uparrow}^{0}(k, q) n_{\downarrow}^{0}\left(k^{\prime}, q\right)}{\sin (k+q / 2)+\sin \left(k^{\prime}+q / 2\right)} .
$$

Substituting $p=k+q / 2, p^{\prime}=k^{\prime}+q / 2$ one obtains

$$
E_{2} / L=-\frac{U^{2}}{(2 \pi)^{3}} \int_{0}^{\pi} d q \frac{1}{2 \sin (q / 2)} J(q),
$$

where

$$
J(q)=4 \int_{0}^{q / 2} \frac{d p d p^{\prime}}{\cos p+\cos p^{\prime}}
$$

Integration over $p^{\prime}$ and the substitution $y=\tan (p /$ $2) \tan (q / 4)$ yields

$$
J(q)=4 \int_{0}^{\tan ^{2}(q / 4)} \frac{d y}{y} \ln \frac{1+y}{1-y} .
$$

We finally substitute $z=\tan ^{2}(q / 4)$ and find

$$
E_{2} / L=-\frac{U^{2}}{4 \pi^{3}} \int_{0}^{1} \frac{d z}{z} \int_{0}^{z} \frac{d y}{y} \ln \frac{1+y}{1-y} .
$$

Using

$$
\ln \frac{1+y}{1-y}=2 \sum_{j=0}^{\infty} \frac{y^{2 j+1}}{2 j+1}
$$

we obtain the result

$$
E_{2} / L=-\frac{U^{2}}{2 \pi^{3}} \sum_{j=0}^{\infty} \frac{1}{(2 j+1)^{3}}=-\frac{7}{16 \pi^{3}} \xi(3) U^{2}
$$

${ }^{1}$ J. Hubbard, Proc. R. Soc. London, Ser. A 276, 238 (1963).

${ }^{2}$ J. Kanamori, Prog. Theor. Phys. 30, 257 (1963).

${ }^{3}$ M. C. Gutzwiller, Phys. Rev. Lett. 10, 159 (1963).

${ }^{4}$ E. H. Lieb and F. Y. Wu, Phys. Rev. Lett. 20, 1445 (1968).

${ }^{5}$ D. R. Penn, Phys. Rev. 142, 350 (1966).

${ }^{6}$ E. N. Economou and P. N. Poulopoulos, Phys. Rev. B 20, 4756 (1979).

${ }^{7}$ M. Takahashi, Prog. Theor. Phys. 44, 348 (1970); 45, 756 (1971).

${ }^{8}$ H. Shiba, Phys. Rev. B 6, 930 (1972).

${ }^{9}$ J. Goldstone, Proc. R. Soc. London, Ser. A 293, 267 (1957).

${ }^{10}$ See, for example, R. D. Mattuck, A Guide to Feynman Diagrams in the Many-Body Problem, 2nd ed. (McGraw-Hill, New York, 1976).

${ }^{11}$ For a half-filled band on a simple-cubic lattice the correlation energy has already been calculated in second-order perturbation theory by L. G. Caron and G. Kemeny, Phys. Rev. B 3, 3007 (1971). Direct comparison with their result is difficult, since these authors did not explicitly present the result for the $U^{2}$ term and only plotted the total ground-state energy for small $U$. Subtracting the Hartree-Fock contribution from their curve, their result for the $U^{2}$ term is consistent with our evaluation.

${ }^{12}$ W. Metzner and D. Vollhardt, Phys. Rev. Lett. 62, 324 (1989).

${ }^{13}$ Note that diagrams with tadpole insertions cancel each other whereas graphs which "contradict the exclusion principle" are to be included (for a detailed discussion, see Ref. 9).

${ }^{14}$ The individual Goldstone diagrams yield positive and negative contributions whose magnitudes are all of the same order. This leads to substantial cancellations between them. We ran the Monte Carlo integration for $100 \mathrm{~h}$ on a personal computer which allows us to estimate the resulting numerical error as about $10 \%$. The exact coefficient in (6) lies within the error bar.

${ }^{15}$ W. Kohn and J. M. Luttinger, Phys. Rev. 118, 41 (1960).

16J. M. Luttinger and J. C. Ward, Phys. Rev. 118, 1417 (1960).

${ }^{17} \mathrm{See}$, for example, The Proceedings of the International Conference on High Temperature Superconductors and Materials and Mechanisms of Superconductivity, Interlaken, Switzerland, 1988, edited by J. Müller and J. L. Olsen (North-Holland, Amsterdam, 1988).

${ }^{18}$ H. J. Schulz, Europhys. Lett. 4, 609 (1987). 